\title{
Effect of temperature on preventing electromigration damage based on increasing threshold current density in a thin metal passivated line
}

\author{
Hiroto IKADAI*, Yasuhiro KIMURA* and Masumi SAKA* \\ *Department of Nanomechanics, Tohoku University \\ Aoba 6-6-01, Aramaki, Aoba-ku, Sendai 980-8579, Japan \\ E-mail: saka@ism.mech.tohoku.ac.jp
}

Received 14 December 2015

\begin{abstract}
The influencing factors associated with the temperature dependence of electromigration (EM) damage are discussed. EM is the atomic diffusion in a metal line when the line is stressed with high current density and causes EM damage called voids and hillocks. Voids are the depletion of the metal atoms and lead to breaking of the lines. Hillocks are the accumulation of the metal atoms and cause short circuits. Passivation covering the metal lines has been used as one of the ways to prevent EM damage because it constrains the formation of hillocks and voids at the same time owing to the conservation of mass. The passivation characteristics such as hardness affect the ability to inhibit EM damage. EM damage is significantly affected by temperature, and to reveal the relation of the passivation hardness with temperature contributes to improving EM reliability. In addition, other factors are thought to affect the temperature dependence of EM damage. In the present paper, we investigate the temperature dependence of EM damage measured by means of threshold length product and clarify several factors affecting the temperature dependence of EM damage. A tetraethyl orthosilicate layer is used as passivation on a conventional Al line-type structure sample. Through the experiments, we demonstrate that EM damage can be prevented much more by decreasing temperature and the increase of the passivation hardness with decreasing temperature is assumed to have an influence on the ability of passivation to prevent EM damage. We also discuss the effective bulk modulus and the electrical resistivity of $\mathrm{Al}$ as the factors affecting the temperature dependence of EM damage.
\end{abstract}

Key words : Electromigration, Passivation, Hardness, Threshold current density, Temperature

\section{Introduction}

The reliability of electronic devices as they become smaller is a big concern. As the cross-sectional area of a thin film line gets smaller and smaller, it causes an increase in the current density and electromigration (EM) damage in the metal lines. EM is the phenomenon of atomic transport in a metal line when the line is stressed with high current density, which causes voids and hillocks. Voids are created by the depletion of atoms and lead to disconnections of lines. Hillocks are created by the accumulation of atoms and cause short circuits of lines. Many researchers have studied ways to prevent EM damage and increase the reliability of electronic devices (Huntington and Grone, 1961 and Huntington, 1975). One way to prevent EM damage is to cover the lines with a passivation layer. As the lines are covered with a thicker passivation layer, the ability to inhibit the formation of hillocks and prevent EM damage increases (Spitzer and Schwartz, 1969, Ainslie, et al., 1972, Blech, 1976 and Lloyd and Smith, 1983). The passivation ability to prevent EM damage was expected to depend on the passivation hardness (Lloyd and Smith, 1983). Another way to reduce EM damage is to decrease the substrate temperature. Black (1969) reported that the median time to failure (MTF) is subject to temperature.

Blech (1976) suggested the existence of a threshold current density $j_{\mathrm{th}}$ : the critical current density value for the start of EM damage. In Blech's study, it was found that $j_{\mathrm{th}}$ is inversely proportional to the line length $l$, whereby the threshold length product $j_{\mathrm{th}} \cdot l$ is used to assess EM damage. In addition, from EM tests using Al line-type samples 
covered with passivation, the relation between $j_{\mathrm{th}} \cdot l$ and temperature was presented, which indicated that $j_{\mathrm{th}} \cdot l$ was lower and there was more EM damage at higher temperature (Blech, 1976 and Atakov, et al., 1994). Many factors are thought to affect the temperature dependence of $j_{\mathrm{th}} \cdot l$.

In the present paper, we discuss the temperature dependence of $j_{\mathrm{th}} \cdot l$ more deeply to improve EM reliability. Since the passivation hardness is predicted to relate to the temperature dependence of $j_{\mathrm{th}} \cdot l$, we investigate the temperature dependence of $j_{\mathrm{th}} \cdot l$ and the passivation hardness through two types of experiment: the EM test and the passivation hardness test. We also discuss the effective bulk modulus $\kappa$ and the electrical resistivity $\rho$ of $\mathrm{Al}$ as the factors affecting the temperature dependence of $j_{\mathrm{th}} \cdot l$. The conventional Al line-type structure sample and a tetraethyl orthosilicate (TEOS) passivation layer are used in this work.

\section{Theoretical background}

The atomic flux induced by EM is generated in the direction of electron flow because of high current stressing. Passivation inhibits the formation of hillocks and voids at the same time owing to the conservation of mass. In a passivated line, the gradient of the hydrostatic stress builds up because the atomic density decreases at the cathode side and increases at the anode side owing to atomic diffusion. This gradient generates backflow, which is the atomic flux in the opposite direction of EM (Blech, 1976). EM damage is prevented because the backflow weakens the atomic flux induced by electron flow. The atomic flux in a passivated line is expressed as follows (Huntington and Grone, 1961, Wever, 1973, Lloyd, et al., 1982, Korhonen, et al., 1993 and Sasagawa, et al., 2002a):

$$
|\mathbf{J}|=\frac{N D_{0}}{k T} \exp \left(-\frac{Q}{k T}\right)\left(\left|Z^{*}\right| e \rho|\mathbf{j}|-\frac{k \Omega}{N_{0}} \frac{\partial N}{\partial l}\right),
$$

where $N$ is the atomic density, $D_{0}$ is the diffusion constant, $k$ is the Boltzmann constant, $T$ is the absolute temperature, $Q$ is the activation energy, $\Omega$ is the atomic volume, $N_{0}$ is the atomic density under stress-free conditions, $Z^{*}(<0$ in the case of $\mathrm{Al}$ ) is the effective valence, $e$ is the charge of an electron, $\mathbf{j}$ is current density vector in the direction of $-\mathbf{J}$ and $\partial N / \partial l$ is an atomic density gradient in the direction of $\mathbf{J}$. The term $\left|Z^{*}\right| e \rho|\mathbf{j}|$ shows the driving force resulting from current stressing, and $\left(\kappa \Omega / N_{0}\right) \partial N / \partial l$ shows the backflow. In the case of $\mathbf{J}=0$, two atomic fluxes induced by the driving force and the backflow are balanced, and no EM damages appear. The gradient $\partial N / \partial l$ is generated after an adequate time and increases with the increase of $|\mathbf{j}|$. When $N$ at the anode side reaches $N_{\max }$, which is the critical atomic density for hillock formation, and $N$ at the cathode side is $N_{\text {min }}$, which is the critical atomic density for void formation, EM damage is generated (Sasagawa, et al., 2002b). For this situation, $|\mathbf{j}|$ is called threshold current density $j_{\mathrm{th}}$. Therefore, at $|\mathbf{j}|=j_{\text {th }}, \partial N / \partial l$ is given by

$$
\frac{\partial N}{\partial l}=\frac{N_{\max }-N_{\min }}{l},
$$

and from Eqs. (1) and (2), $j_{\text {th }}$ is given as

$$
j_{\mathrm{th}}=\frac{\kappa \Omega}{N_{0}\left|Z^{*}\right| e \rho} \frac{N_{\max }-N_{\min }}{l} .
$$

Equation (3) shows that $j_{\text {th }}$ is inversely proportional to $l$, so $j_{\text {th }} \cdot l$ can be used to assess EM damage. It is predicted that $N_{\max }-N_{\min }$ increases owing to the increase of the passivation hardness. Hence, $j_{\mathrm{th}} \cdot l$ will increase with the increase of the passivation hardness and EM resistance is expected to be improved.

\section{Experimental procedure}

In the present study, we performed two types of experiment: one for examining the effect of temperature on $j_{\mathrm{th}} \cdot l$ and another for examining the relationship between the passivation hardness and the substrate temperature. The EM test sample was the Al line-type structure covered with a passivation layer, as shown in Fig. 1(a). The enlarged 
illustration of the sample at the cathode side is shown in Fig. 1(b). Figure 1(c) shows the cross-sectional view of the sample. A Si wafer was oxidized to form a $300 \mathrm{~nm}$ thick $\mathrm{SiO}_{2}$ layer. TiN and $\mathrm{Al}$ were sequentially sputtered to form 300 $\mathrm{nm}$ thick (deposition for $18 \mathrm{~min}$ at $473 \mathrm{~K}$ ) and $570 \mathrm{~nm}$ thick layers (deposition for $5 \mathrm{~min} 45 \mathrm{~s}$ at room temperature), respectively. Stripes $500 \mu \mathrm{m}$ in length and $9 \mu \mathrm{m}$ in width were formed by etching Al and TiN layers using photolithography, and a $10 \mu \mathrm{m}$ slit was formed by etching Al. The TEOS layer, which was $780 \mathrm{~nm}$ thick, was deposited as passivation on the sample using chemical vapor deposition (CVD) (deposition for $6 \mathrm{~min} 40 \mathrm{~s}$ at $623 \mathrm{~K}$ ). The passivation layer was etched to form a conductive pad to apply current and measure voltage.

The electron passes through the sample from the cathode to the anode during current stressing, as shown in Fig. 1(c). At the slit, electrons flow in the TiN line. Since the electrical resistivity of $\mathrm{Al}$ is approximately one twentieth that of TiN, electrons pass preferentially to the $\mathrm{Al}$ line. In the $\mathrm{Al}$ line, $\mathrm{Al}$ atoms are diffused in the direction of electron flow, and the accumulation and depletion of $\mathrm{Al}$ atoms occur at the anode side and at the cathode side, respectively.

The product $j_{\mathrm{th}} \cdot l$ can be calculated through the drift velocity measurement, which has been reported by Blech (1976). The EM test was carried out at various substrate temperatures in the range of 473 to $663 \mathrm{~K}$ to examine the temperature dependence of $j_{\mathrm{th}} \cdot l$. Appropriate values of current density were used to reduce the test time.

In the passivation hardness test, on the other hand, a TEOS layer was deposited as passivation on a Si wafer using CVD. The passivation hardness was measured at various values of temperature using nanoindenter (Hysitron TI 950 TriboIndenter). The indentation hardness of passivation $H$ is defined as follows (Oliver and Pharr, 1992):

$$
H=\frac{P_{\max }}{A},
$$

where $P_{\max }$ is maximum load and $A$ is projected contact area of the hardness impression. The passivation layer thickness was $1,500 \mathrm{~nm}$, which was adequate for using nanoindentation (the indentation depth was less than $180 \mathrm{~nm}$ ). In the test, a diamond tip of four-sided pyramid shape was used.
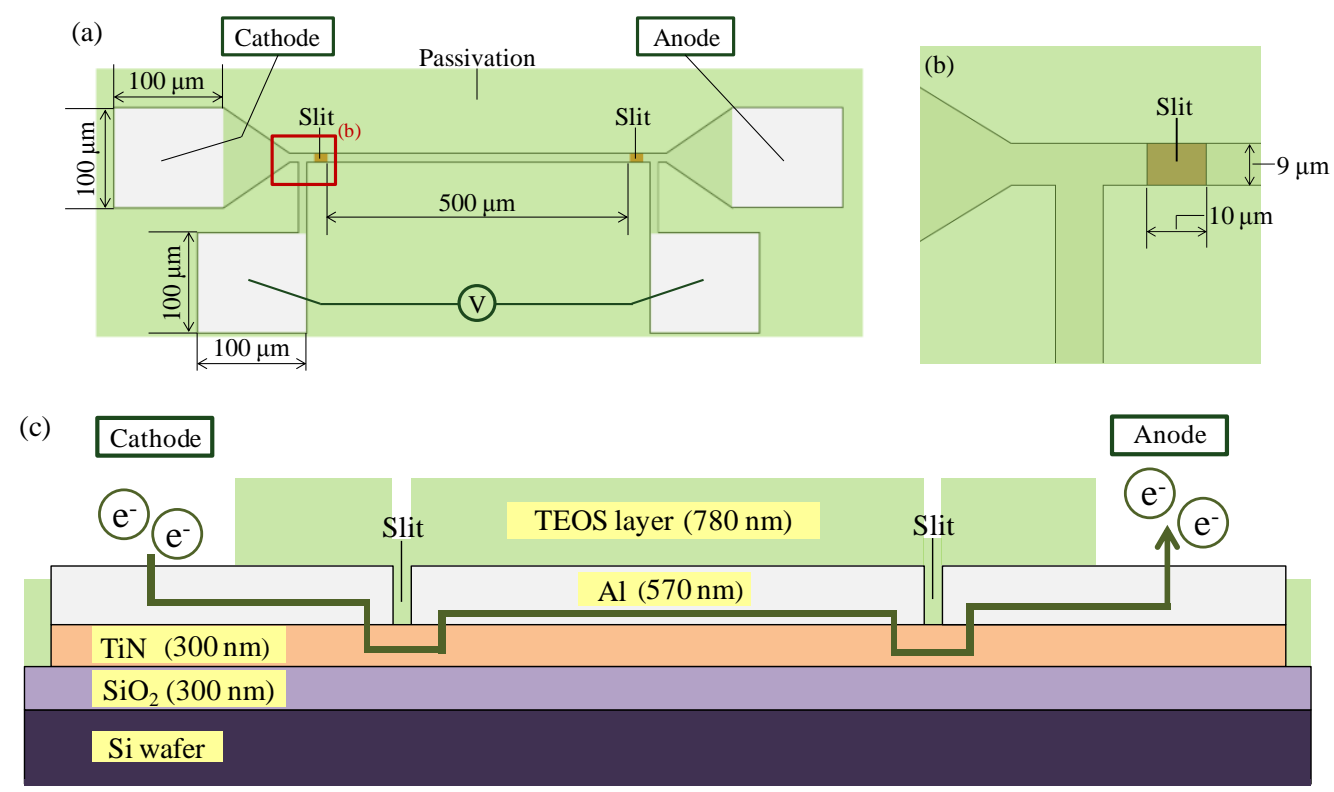

Fig. 1 Illustrations of the EM test sample: (a) the whole image of the sample, (b) the enlargement of the sample at the cathode side and (c) the cross-sectional view of the sample.

\section{Results and discussion}

\section{4-1. The measurement of $j_{\mathrm{th}} \cdot l$}

In the EM test, voids and hillocks were formed at the cathode side and anode side of the Al line, respectively. The enlarged optical microscope image at the cathode side before the supply of current is shown in Fig. 2(a), and after the 
supply of current is shown in Fig. 2(b). The drift velocity was evaluated based on the measurement of the void area. The average drift velocity $v$ is theoretically expressed as follows (Huntington and Grone, 1961, Wever, 1973, Blech, 1976, Lloyd, et al., 1982, Korhonen, et al., 1993 and Sasagawa, et al., 2002a):

$$
v=\frac{|\mathbf{J}|}{N}=\frac{D_{0}}{k T} \exp \left(-\frac{Q}{k T}\right)\left(\left|Z^{*}\right| e \rho|\mathbf{j}|-\frac{\kappa \Omega}{N_{0}} \frac{\partial N}{\partial l}\right) .
$$

Figure 3 shows the relation of $v$ and $|\mathbf{j}|$ obtained by the present EM test and the error bars indicate the standard deviation, where 4 or 5 measurements were carried out at each value of substrate temperature and current density. Since $v$ is proportional to $|\mathbf{j}|$ in Eq. (5), the approximation line for $v$ and $|\mathbf{j}|$ relation is assumed to be straight. As shown in Fig. $3, j_{\text {th }}$ was obtained by extrapolating a line to zero drift velocity. The relation of $j_{\mathrm{th}} \cdot l$ and the substrate temperature is shown in Fig. 4 . The product $j_{\mathrm{th}} \cdot l$ increased with decreasing the substrate temperature. Therefore, the resistance to EM can be increased much more by decreasing the temperature.

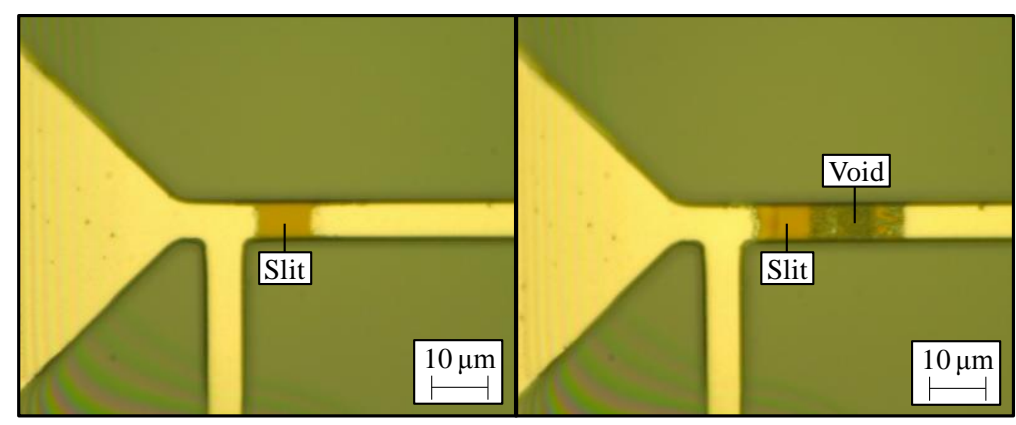

(a)

(b)

Fig. 2 The enlarged optical microscope images at the cathode side: (a) before current supply and (b) after current supply.

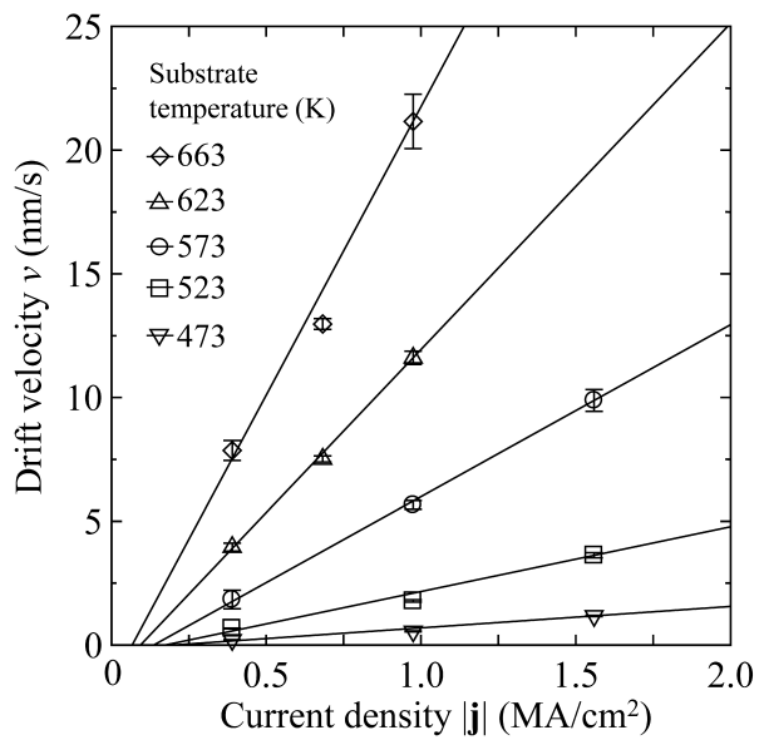

Fig. 3 The measured drift velocity as a function of current density. 


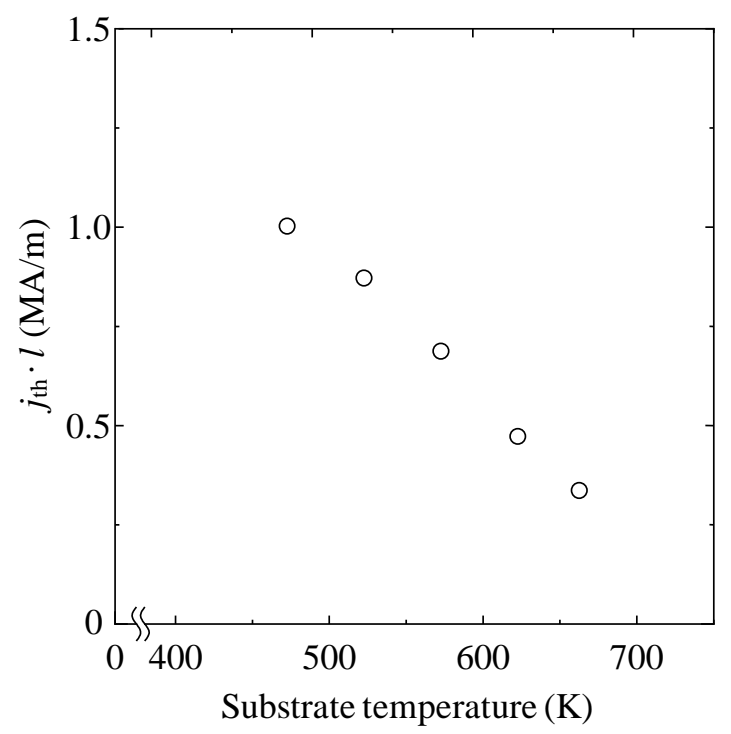

Fig. 4 The relation of $j_{\mathrm{th}} \cdot l$ and the substrate temperature.

\section{4-2. The temperature dependence of $j_{\mathrm{th}} \cdot l$}

For improving EM resistance, the temperature should be decreased as stated above. The clarification of the parameters affecting the temperature dependence of $j_{\mathrm{th}} \cdot l$ contributes to improving EM resistance. In this work, we do not treat the change in diffusion mode with temperature based on the premise described below. Equation (5) is transformed into

$$
\ln (v T)=-\frac{Q}{k T}+\ln \left\{\frac{D_{0}}{k}\left(\left|Z^{*}\right| e \rho|\mathbf{j}|-\frac{\kappa \Omega}{N_{0}} \frac{\partial N}{\partial l}\right)\right\} .
$$

Equation (6) can be used to obtain $Q$ from the usual Arrhenius plot. An example of the Arrhenius plot at $|\mathbf{j}|=0.4$ $\mathrm{MA} / \mathrm{cm}^{2}$ is shown in Fig. 5. The measured average value of $Q$ is about $0.56 \mathrm{eV}$ in $473-663 \mathrm{~K}$ and agrees with that for grain boundary diffusion which has been reported (Blech, 1976 and Tan and Roy, 2007). The value of $Q$ invariable in the treated temperature range implies that the diffusion mode does not change.

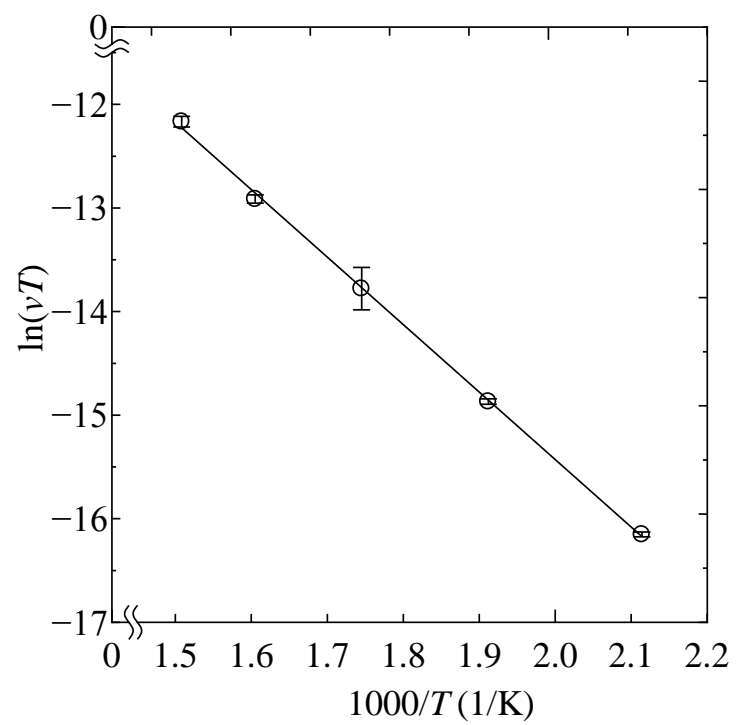

Fig. 5 An example of the Arrhenius plot at $|\mathbf{j}|=0.4 \mathrm{MA} / \mathrm{cm}^{2}$. 
To clarify the factors affecting the temperature dependence of $j_{\mathrm{th}} \cdot l$, we discuss the parameters which depend on temperature in Eq. (3). Figure 6(a) shows the dependence of $H$ on the substrate temperature. An example of the relation between load and indentation displacement at $575 \mathrm{~K}$ is shown in Fig. 6(b). In the passivation hardness test, we carried out 4 or 5 measurements at each value of temperature. As shown in Fig. 6(a), $H$ increased with decreasing the substrate temperature. The increase of $H$ may improve the ability to inhibit the formation of hillocks with increasing $N_{\max }-N_{\min }$ in Eq. (3), which indicates that the increase of $H$ increases $j_{\text {th }} \cdot l$. Thus, $H$ is assumed to have an influence on preventing EM damage.
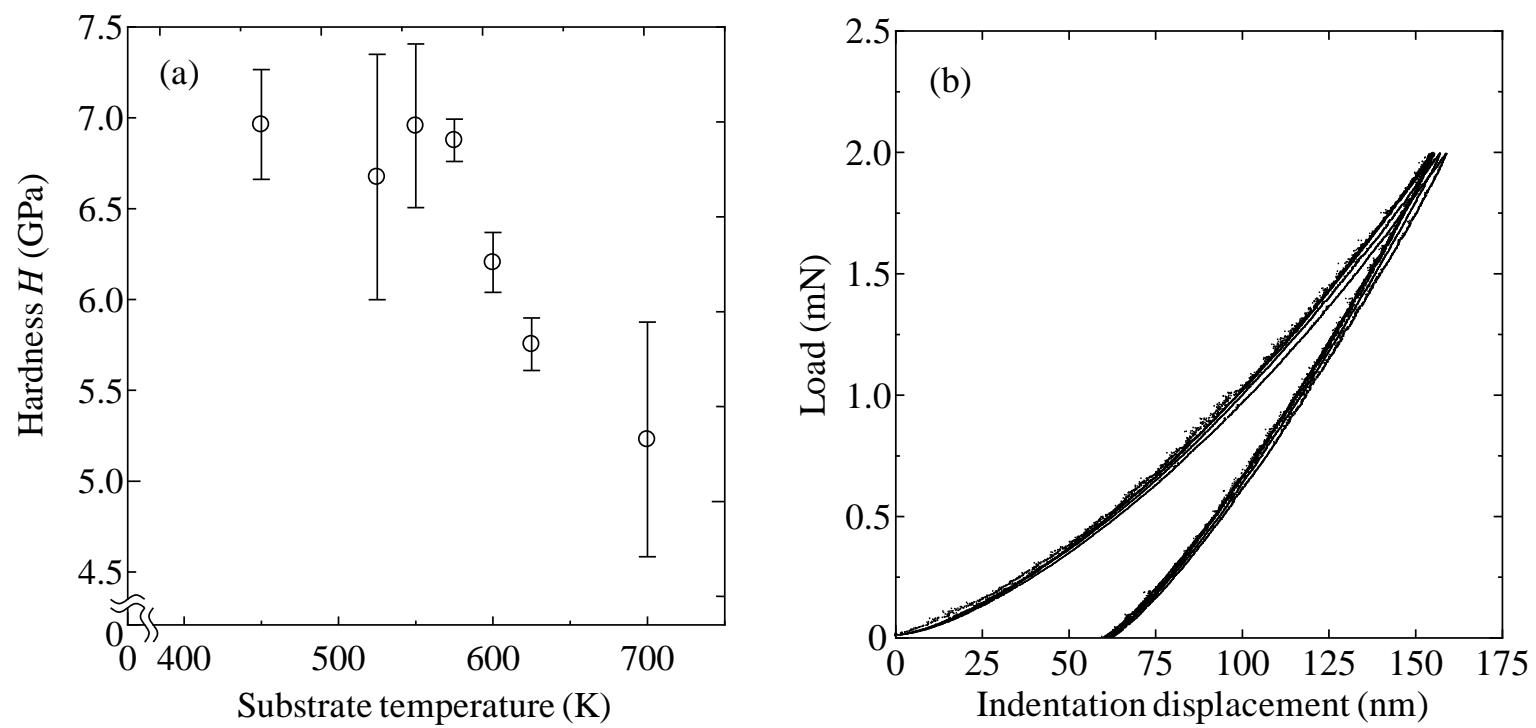

Fig. 6 The passivation hardness test using nanoindentation: (a) the dependence of the passivation hardness on the substrate temperature and (b) an example of the relation between load and indentation displacement at $575 \mathrm{~K}$.

In addition, we need to consider the temperature dependence of $\kappa$ and $\rho$. It has been reported that $\kappa$ increases with decreasing temperature (Gerlich and Fisher, 1969) and $\rho$ decreases with decreasing temperature (Desai, et al., 1984). From Eq. (3), the reasons why $j_{\text {th }} \cdot l$ increased with decreasing temperature include the increase of $\kappa$ and the decrease of $\rho$ with decreasing temperature.

\section{Conclusions}

The temperature dependence of the threshold length product $j_{\mathrm{th}} \cdot l$, which has been used to assess EM damage, was investigated through EM tests. In addition, the passivation hardness $H$, the effective bulk modulus $\kappa$ and the electrical resistivity $\rho$ of $\mathrm{Al}$, which depend on temperature, were discussed to clarify the details of the temperature dependence of $j_{\text {th }} \cdot l$. A tetraethyl orthosilicate (TEOS) layer on a conventional Al line-type structure sample was used as passivation. The product $j_{\mathrm{th}} \cdot l$ increased with decreasing the substrate temperature. Using nanoindentation, $H$ was found to increase with decreasing the substrate temperature. Also, the reasons why $j_{\mathrm{th}} \cdot l$ increased with decreasing temperature include the increase of $\kappa$ and the decrease of $\rho$ with decreasing temperature. Therefore, EM damage can be prevented much more by decreasing temperature which significantly affects $H, \kappa$ and $\rho$.

\section{Acknowledgements}

This work was supported by JSPS KAKENHI Grant-in-Aid for Scientific Research (B) No. 26289001. This work was performed at the Micro/Nano-Machining Research and Education Center of Tohoku University. This work was also performed at the FEEMA facilities of OASIS, Muroran Institute of Technology supported by the MEXT Project for Creation of Research Platforms and Sharing of Advanced Research Infrastructure. 


\section{References}

Ainslie, N.G., d'Heurle, F.M. and Wells, O.C., Coating, mechanical constraints, and pressure effects on electromigration, Applied Physics Letters, Vol.20, No.4 (1972), pp.173-174.

Atakov, E.M., Clement, J.J. and Miner, B., Two electromigration failure modes in polycrystalline aluminum interconnects, Proceedings of the 32nd Annual IEEE International Reliability Physics (1994), pp.213-224.

Black, J.R., Electromigration failure modes in aluminum metallization for semiconductor devices, Proceedings of the IEEE, Vol.57, No.9 (1969), pp.1587-1594.

Blech, I.A., Electromigration in thin aluminum films on titanium nitride, Journal of Applied Physics, Vol.47, No.4 (1976), pp.1203-1208.

Desai, P.D., James, H.M. and Ho, C.Y., Electrical resistivity of aluminum and manganese, Journal of Physical and Chemical Reference Data, Vol.13, No.4 (1984), pp.1131-1172.

Gerlich, D. and Fisher, E.S., The high temperature elastic moduli of aluminum, Journal of Physics and Chemistry of Solids, Vol.30, No.5 (1969), pp.1197-1205.

Huntington, H.B. and Grone, A.R., Current-induced marker motion in gold wires, Journal of Physics and Chemistry of Solids, Vol.20, Nos.1-2 (1961), pp.76-87.

Huntington, H.B., in Diffusion in solids: Recent development (Nowick, A.S., Burton, J.J., eds.) (1975), pp.303-352, Academic Press Inc., New York.

Korhonen, M.A., Børgesen, P., Tu, K.N. and Li, C.-Y., Stress evolution due to electromigration in confined metal lines, Journal of Applied Physics, Vol.73, No.8 (1993), pp.3790-3799.

Lloyd, J.R., Smith, P.M. and Prokop, G.S., The role of metal and passivation defects in electromigration-induced damage in thin film conductors, Thin Solid Films, Vol.93, Nos.3-4 (1982), pp.385-395.

Lloyd, J.R. and Smith, P.M., The effect of passivation thickness on the electromigration lifetime of $\mathrm{Al} / \mathrm{Cu}$ thin film conductors, Journal of Vacuum Science and Technology A, Vol.1, No.2 (1983), pp.455-458.

Oliver, W.C. and Pharr, G.M., An improved technique for determining hardness and elastic modulus using load and displacement sensing indentation experiments, Journal of Materials Research, Vol.7, No.6 (1992), pp.1564-1583.

Sasagawa, K., Hasegawa, M., Saka, M. and Abé, H., Governing parameter for electromigration damage in the polycrystalline line covered with a passivation layer, Journal of Applied Physics, Vol.91, No.4 (2002a), pp.1882-1890.

Sasagawa, K., Hasegawa, M., Saka, M. and Abé, H., Prediction of electromigration failure in passivated polycrystalline line, Journal of Applied Physics, Vol.91, No.11 (2002b), pp.9005-9014.

Spitzer, S.M. and Schwartz, S., The effects of dielectric overcoating on electromigration in aluminum interconnections, IEEE Transactions on Electron Devices, Vol.16, No.4 (1969), pp.348-350.

Tan, C.M. and Roy, A., Electromigration in ULSI interconnects, Materials Science and Engineering R, Vol.58, Nos.1-2 (2007), pp.1-75.

Wever, H., in Elektro-und thermotransport in metallen (1973), pp.30-39, Johann Ambrosius Barth, Leipzig. 